Proceedings of the WELCOME Scientific Meeting on Hybrid Nanostructures, Torun, Poland, August 28-31, 2011

\title{
Energy Transfer in Peridinin-Chlorophyll-Protein-Metallic Nanoparticle Hybrid Nanostructure: Effect of Spatial Arrangement
}

\author{
M.K. SChmidT AND S. MaCKOWSKI* \\ Optics of Hybrid Nanostructures Group, Institute of Physics, Nicolaus Copernicus University \\ Grudziądzka 5/7, 87-100 Toruń, Poland
}

\begin{abstract}
We theoretically investigate dynamics of excitation in a hybrid nanostructure comprising a photosynthetic complex peridinin-chlorophyll-protein (PCP) coupled to a gold spherical nanoparticle. Our model includes the analytical description of radiative and non-radiative relaxation channels of the chlorophylls in PCP, as well as the change of energy transfer rate within the PCP due to the presence of metal scatterer. We show that by measuring the intensity of fluorescence from selectively excited chlorophyll molecules in the PCP complex, elementary geometric properties of the system can be deduced.
\end{abstract}

PACS: 33.50.-j, 73.20.Mf, 82.20.Rp

\section{Introduction}

Metallic nanoparticles, due to their unique ability to manipulate light at the nanoscale and the simplicity of spectral tuning of the plasmon resonance, have been a subject of intense studies in various research fields, including quantum optics [1, 2], sensor development [3], and photovoltaics [4]. Most of these studies focus on nanofabrication of advanced plasmonic nanostructures with well-defined spectroscopic properties, as well as on integrating metallic nanoparticles with inorganic materials, such as quantum dots or fluorescent molecules. In the latter case, it has been demonstrated that, depending upon the geometry of the metallic nanoparticle-dye assembly, either efficient quenching of the fluorescence or strong enhancement of the emission can be achieved. One of the key parameters determining the actual outcome is the separation between the elements comprising the hybrid [5]. It has been shown that the optimal distance for the enhancement of emission ranges between 10 and $25 \mathrm{~nm}$, and the enhancement factors of up to several tens has been observed [5,6]. Much less attention has been paid to more complex systems, comprising more than one chromophore. As soon as the optical properties of a molecular assembly that is to be attached to the metallic nanoparticle promote resonant energy transfer, the analysis of the experimental results as well as modeling thereof becomes considerably more difficult [7-9].

Recently, several groups have attempted to couple biologically relevant systems, such as photosynthetic complexes, with various types of metallic nanostructures [10-13]. The motivation behind these efforts was to investigate the effects of plasmon excitations on the absorption

* corresponding author; e-mail: mackowski@fizyka.umk.pl efficiency and, presumably, energy transfer dynamics in these complicated, multichromophoric systems. It has been shown that the fluorescence of chlorophylls within such complexes can be strongly enhanced, mainly due to an increase of the absorption rate of the light-harvesting pigments [14]. Furthermore, such complexes exhibit a very well defined geometry of the pigments thanks to the protein scaffold that binds them together. Therefore, they provide an attractive system for studying the influence of plasmon excitations on the energy transfer dynamics.

\section{Peridinin-chlorophyll-protein}

Our model system is a peridinin-chlorophyll-protein (PCP) complex reconstituted with chlorophyll $a(\mathrm{Chl} a)$ and chlorophyll $b[15,16]$. The structure of PCP complex from dinoflagellate Amphidinium carterae has been determined by Hofmann et al. [17] with a resolution better than $1.5 \AA$. In its native form it consists of three monomers forming a trimer, with each monomer binding two Chl $a$ and eight peridinin (Per) molecules arranged in two almost symmetric clusters. It has been demonstrated that the apoprotein of the PCP can be reconstituted with various Chls [15] including mixtures of Chls, while preserving the overall structure of the refolded protein.

Absorption of the PCP complex exhibits two spectrally separated regions - a strong and broad band between 400 and $550 \mathrm{~nm}$ [18] due to Per absorption and a narrow $Q_{Y}$ transition band of the chlorophyll. Furthermore, chlorophylls contribute to the absorption spectrum through a Soret band at $400 \mathrm{~nm}$. As we are interested in a modification of the excitation dynamics in a simplified system comprising chlorophylls exclusively, we focus on the interactions between the $Q_{Y}$ transitions within a single monomer or PCP. The energies of these transitions differ between various chlorophyll molecules, with 
the absorption of Chl $a$ and $\mathrm{Chl} b$ centered at around 660 and $640 \mathrm{~nm}$, respectively and the fluorescence red-shifted by around $10 \mathrm{~nm}$. Previous studies, carried on both ensemble and single-molecule levels [11] revealed that this particular pigment combination features a bidirectional energy transfer, both from $\mathrm{Chl} a$ to $\mathrm{Chl} b$, and from $\mathrm{Chl} b$ to Chl $a$. The efficiencies of these two energy transfer channels are comparable.

Consequently, this water-soluble photosynthetic complex can be considered a model system for studying plasmon-induced effects. In comparison to other pigment-protein complexes, such as photosystem I or photosystem II, which contain tens or hundreds of chlorophylls, both the structure and the pigment composition in PCP are relatively simple. Furthermore, the apoprotein of the PCP complex can be easily reconstituted with mixtures of chlorophylls thus providing a tool to engineer the energy transfer dynamics between pigments in a well-defined protein geometry. Last but not least, the absorption of the PCP complex matches well with the plasmon frequencies of both gold and silver small spherical nanoparticles $[19,20]$.

\section{Model}

Our analysis is based on a simplified model of a heterogeneous pair of chlorophylls, with the geometry inherited from the PCP complex: the relative orientation and spectral properties of molecules are identical to those of chlorophylls embedded in the protein scaffold. The pair of chlorophylls is positioned in the vicinity of a spherical gold nanoparticle (MNP) characterized by the complex dielectric function [21]. We investigate the dependence of the excitation dynamics within such a hybrid complex on the orientation of PCP, its distance to the surface of metal, and a radius of the nanoparticle.

In order to quantitatively describe the influence of the plasmon excitation on the spectroscopic properties of a photosynthetic complex, an accurate description of all the relaxation pathways for each molecule is required. It has been recently shown that the fluorescence of individual chromophores in the PCP complex as well as the energy transfer - both between each pair of chlorophylls and from the molecules to the metallic nanoparticle can be accurately described by considering the exact electric field distribution within the complex [8, 9, 22]. Below we give a short description of all the relevant mechanisms.

\subsection{Radiation of the electric dipole}

In order to evaluate the rate at which energy is radiated away from each of the chlorophyll molecules in the hybrid nanostructure, we decouple the problem and separately investigate two simplified subsystems. Each one comprises a single chlorophyll molecule with a fixed electric dipolar transition $\left(Q_{Y}\right)$ moment $\boldsymbol{\mu}$ in the vicinity of a spherical metallic nanoparticle. By calculating the energy flux of the radiation from such a system, and normalizing it to the radiation power of a classical electric dipole in a free space, an enhancement of the radiative decay rate can be calculated. This problem has been addressed analytically, with the extended Mie theory [6, 23], for two orientations of the dipole $\boldsymbol{\mu}$ - perpendicular (with the enhancement ratio denoted as $\left.\Gamma_{\perp}^{\mathrm{rad}}\right)$ and parallel $\left(\Gamma_{\|}^{\mathrm{rad}}\right)$ to the surface of the MNP. These values can be applied to calculate the rate of radiation for any orientation of the dipole

$$
\Gamma^{\mathrm{rad}}(\alpha) \approx \sin ^{2}(\alpha) \Gamma_{\|}^{\mathrm{rad}}+\cos ^{2}(\alpha) \Gamma_{\perp}^{\mathrm{rad}},
$$

where the parameter $\alpha$ is the angle between $\mu$ and the axis $\hat{z}$ connecting a center of the MNP and the dipole.

\subsection{Energy transfer from the molecule to the $M N P$}

Since the electric field generated by a radiating dipole penetrates the metallic nanoparticle, it creates a pathway for efficient nonradiative relaxation. Ohmic losses become important especially in systems where the molecule is close to the surface of the MNP - at small separation distances the nonradiative process dominates an increase of the radiative rate resulting in quenching of the fluorescence. The efficiency of this process can be analytically investigated by calculating the intensity of the electric field inside the particle. For that purpose, the extension of the Mie theory can be used as well, yielding the nonradiative processes rates for two orientation of the dipole with respect to the surface of the metal - perpendicular $\Gamma_{\perp}^{\text {nonrad }}$ and parallel $\Gamma_{\|}^{\text {nonrad }}$. An equation analogous to (1) holds in that case

$$
\Gamma^{\text {nonrad }}(\alpha) \approx \sin ^{2}(\alpha) \Gamma_{\|}^{\text {nonrad }}+\cos ^{2}(\alpha) \Gamma_{\perp}^{\text {nonrad }} .
$$

The rate of all the nonradiative processes contributing to the decay of the excitation can be then calculated as

$$
k^{\text {nonrad }}(\alpha)=\Gamma^{\text {nonrad }}(\alpha) q k_{0}+(1-q) k_{0},
$$

where $q$ is the quantum yield of each of the chlorophylls in the absence of its counter-partner or the MNP and $k_{0}$ is its fluorescence rate.

\subsection{Energy transfer between the chlorophylls}

Last but not least, the most effective and the fastest of mechanisms determining the excitation dynamics in a PCP complex coupled to a gold nanoparticle is the effect of plasmon-enhanced resonance energy transfer between the chlorophylls. To describe this process, we use a formalism of the Förster resonance energy transfer (FRET) [24] based on a dipole-dipole Coulomb interaction between two molecules. FRET occurs when one of the molecules (donor) exhibits an emission spectrum overlapping with the absorption spectrum of the other one (acceptor). It can be further shown that the rate of the energy transfer is proportional to the energy of the acceptor dipole placed in an electric field generated by a donor molecule [8, 22]:

$$
k^{\mathrm{FRET}} \propto\left|\boldsymbol{\mu}_{a} \boldsymbol{E}_{d}\left(\boldsymbol{r}_{a}\right)\right|^{2} .
$$

The above relation is valid also for interacting dipoles in an inhomogeneous environment. Thus, in a situation 
where due to the presence of the MNP the electric field from the donor at the position of the acceptor is changed, the rate of energy transfer is modified by a factor

$$
\Gamma_{d \rightarrow a}^{\mathrm{FRET}}=\frac{\left|\boldsymbol{\mu}_{a} \boldsymbol{E}_{d, \mathrm{MNP}}\left(\boldsymbol{r}_{a}\right)\right|^{2}}{\left|\mu_{a} \boldsymbol{E}_{d, \text { no MNP }}\left(\boldsymbol{r}_{a}\right)\right|^{2}} .
$$

As the distances between all the constituents of the system are much smaller, then the wavelength of radiation $\lambda$, we implement an electrostatic analytical solution to the problem of electrostatic scattering of an electric dipolar field [25].

\section{Results and discussion}

\subsection{Fluorescence intensity and the modification of lifetime}

In a typical optical experiment, two important quantities are determined: the intensity and lifetime of fluorescence from a PCP-MNP hybrid nanostructure. Using the approach described here we obtain results that can be directly compared to such a set of experimentally measured parameters. To relate these quantities to previously defined enhancement factors, we solve the set of rate equations which determine the time dependence of the probability of the excitation of each of the chlorophylls $P_{i}$ :

$$
\begin{aligned}
& \frac{\mathrm{d} P_{1}}{\mathrm{~d} t}=-\left(k_{1}^{\mathrm{nonrad}}+k_{0,1}^{\mathrm{rad}} \Gamma_{1}^{\mathrm{rad}}+k_{1 \rightarrow 2}^{\mathrm{FRET}} \Gamma_{1 \rightarrow 2}^{\mathrm{FRET}}\right) P_{1} \\
& \quad+k_{2 \rightarrow 1}^{\mathrm{FRET}} \Gamma_{2 \rightarrow 1}^{\mathrm{FRET}} P_{2}, \\
& \frac{\mathrm{d} P_{2}}{\mathrm{~d} t}=-\left(k_{2}^{\mathrm{nonrad}}+k_{0,2}^{\mathrm{rad}} \Gamma_{2}^{\mathrm{rad}}+k_{2 \rightarrow 1}^{\mathrm{FRET}} \Gamma_{2 \rightarrow 1}^{\mathrm{FRET}}\right) P_{2} \\
& \quad+k_{1 \rightarrow 2}^{\mathrm{FRET}} \Gamma_{1 \rightarrow 2}^{\mathrm{FRET}} P_{1}
\end{aligned}
$$

with initial unitary excitation probabilities of either of the chlorophyll molecules. Experimentally, this condition corresponds to a $\pi$-pulse excitation of chlorophyll by a laser with wavelength adjusted to the absorption of one of the chlorophylls. The symmetry in the above equations reflects the fact that we account for a bidirectional FRET between the chlorophylls.

The intensity of fluorescence from each of the chlorophyll molecules can be calculated as

$$
I_{i}=k_{0, i}^{\mathrm{rad}} \Gamma_{i}^{\mathrm{rad}} \int_{0}^{\infty} P_{i}(\tau) \mathrm{d} \tau .
$$

The lifetime of fluorescence can be obtained by fitting an exponential decay to the $P$ functions.

\subsection{Geometry of the system}

In order to quantitatively describe the excitation dynamics within our hybrid nanostructure, an accurate parametrization of the geometry is essential. On the other hand, the ability to experimentally discriminate between these geometries is limited by the biochemical methods of coupling the components that form a nanostructure. Therefore, we choose 3 parameters of the system - two of which should be experimentally controllable: the radius of a gold nanoparticle $R$ and distance between the PCP complex and the center of the nanoparticle $d$. The remaining parameter is the orientation of the PCP complex with respect to the surface of the metal. To discretize this property, we consider 4 orientations, as shown in Fig. 1a. The orientations of the chlorophylls and their electric dipolar transition moments within the protein scaffold were taken from [26].

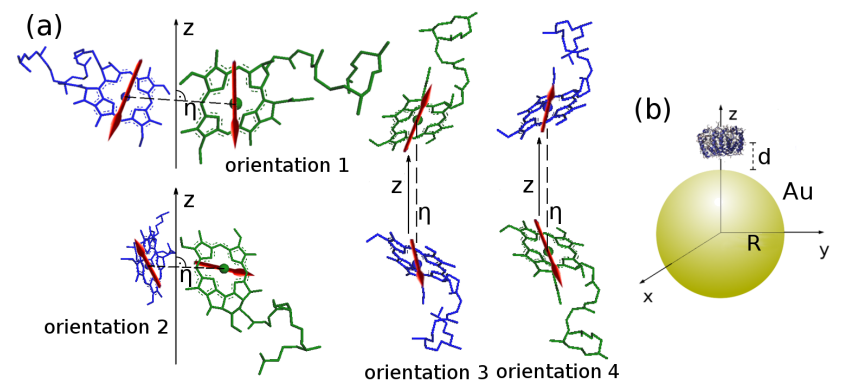

Fig. 1. Selected orientations of chlorophyll molecules embedded in the PCP complex (a) and the schematics of the nanostructure (b). Relative orientations of chlorophylls are shown with respect to the axis $\hat{\boldsymbol{z}}$ connecting the geometric centers of the protein and the gold spherical nanoparticle. Red arrows indicate the orientation of respective dipolar transition moments. In orientations labeled as 1 and 2 ( 3 and 4 ) vector $\boldsymbol{\eta}$ between magnesium atoms in chlorophylls is parallel (perpendicular) to the surface of the metal. Orientation 2 can be obtained from 1 by a $\pi / 2$ rotation about $\boldsymbol{\eta}$, while in orientations 3 and 4 vectors $\boldsymbol{\eta}$ are anti-parallel. The structure of the protein (with the scaffold omitted for the sake of clarity) and the electric dipolar transition moments of chlorophylls were taken from [26]. The orientation of the PCP complex in the schematics of the system (b) is arbitrary.

Our calculations yield the enhancement factors of both energy transfer rates and energy dissipation rates, instead of the actual rates of energy transfer or dissipation. Therefore, in order to calculate the actual dynamics of excitation, which translates to relative intensities of chlorophyll $a$ and chlorophyll $b$, we include experimentally measured values of the fluorescence lifetime, its quantum yield and characteristic times of energy transfer [11] obtained for PCP in the absence of metallic nanoparticles. The photophysical parameters of the two chlorophylls comprising the PCP complex were assumed to be the same as measured for homochlorophyllous PCP complexes [11].

\subsection{Results}

Our analysis reveals a very strong dependence of experimentally accesible parameters on the geometric properties of the hybrid nanostructure. In Fig. 2, an intensity of the fluorescence from a primarily excited chlorophyll molecule, normalized to its free-space value, is plotted as a function of varying MNP-PCP distance (Fig. 2a) or MNP radius (Fig. 2b). As expected, for small moleculemetal distances, fluorescence is efficiently quenched for all four geometries (Fig. 2a). However, for a fixed dis- 
tance of the PCP to the surface of the MNP, two sets of geometries $(1,2$ and 3,4$)$ reveal a qualitatively different dependence on the radius of MNP (Fig. 2b). We believe that this discrepancy can be attributed to the difference in radiative properties of the systems. In the first two geometries, the transition dipoles are primarily oriented perpendicularly to the surface of the MNP and induce a weakly radiating superposition of modes. For the remaining orientations, dipoles are parallel to the interface and, especially for the larger radii, couple into a strongly radiating system. These findings are confirmed by analysis of the enhancement of the radiative processes (not shown here).

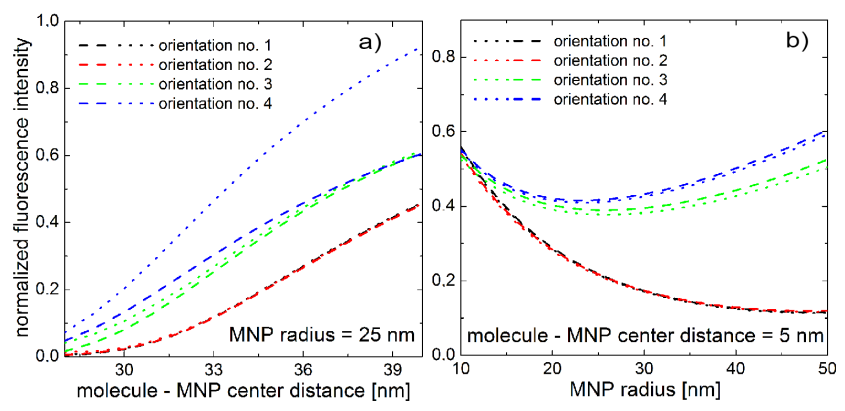

Fig. 2. Intensity of fluorescence from Chl $a$ (dotted lines) and Chl $b$ (dashed lines) as a function of the $\mathrm{MNP}-\mathrm{PCP}$ distance $(\mathrm{MNP}$ radius $=25 \mathrm{~nm}$ ) (a) and MNP radius (molecule-MNP center distance $=5 \mathrm{~nm}$ ) (b) normalized to their free-space values. In both figures, we present the intensity of fluorescence from the initially excited molecule. In (a) the quenching of fluorescence resulting from ohmic losses in MNP is clearly visible in all considered geometries.

In Fig. 3, we plot the dependence of the fluorescence intensity ratio of the two chlorophyll emission. In principle, this ratio is an experimental measure of the efficiency of energy transfer process. For small distances between the PCP and the surface of the metal, in orientations 1 and 2, with both molecules positioned at the same distance from surface of MNP, a strong suppression of resonance energy transfer rate is observed (inset in Fig. 3a), resulting in decoupling of the chlorophylls system (Fig. 3a). For orientations 3 and 4, even though the transfer of energy is slightly enhanced, the nonradiative losses suppress the ability of FRET to equilibrate the excitation of both chlorophylls and - as in previously studied geometries - a strongly asymmetric fluorescence distribution is observed (Fig. 3a). In one specific orientation 3 , in which Chl $a$ is positioned further from the MNP, the fluorescence from this chlorophyll appears to be promoted.

A similar analysis conducted for the fixed MNP-PCP distance (Fig. 3b) does not reveal any strong dependence of the system dynamics on the radius of the MNP. This is probably due to the fact that for small molecule-metal distances, excitation transfer to the nanoparticle does not depend on its radius.

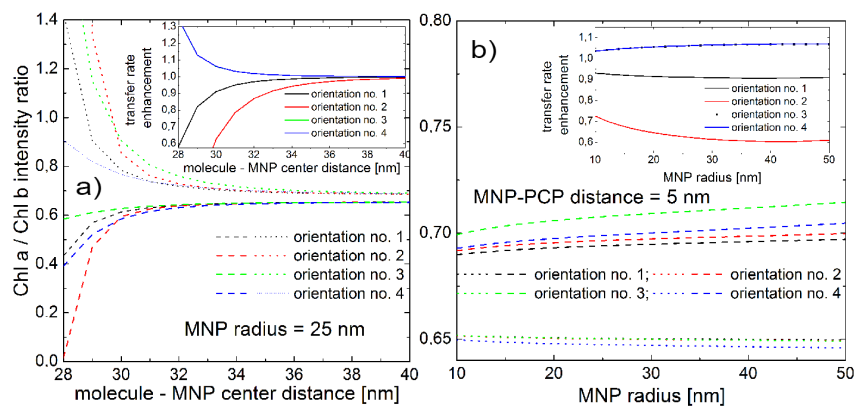

Fig. 3. Ratio of fluorescence from $\mathrm{Chl} a$ and $\mathrm{Chl} b$ as a function of MNP-PCP distance (MNP radius $=25 \mathrm{~nm}$ ) (a) and MNP radius (MNP-PCP distance $=5 \mathrm{~nm})(\mathrm{b})$. The insets show the enhancement of resonance transfer rate in the considered geometries - the results for orientations 3 and 4 are indistinguishable.

It is worth noting that, although separately discussed mechanisms of radiative decay and energy transfer between molecules yield a very different behavior for two sets of geometries discussed in this work, the intensity and ratio of fluorescence - two parameters which can be measured experimentally — reveal similar results for all considered orientations.

\section{Summary}

We have investigated the excitation dynamics in a system comprising a heterochlorophyllous PCP and a gold spherical nanoparticle for 4 different geometries of the setup and varying key parameters - radius of the nanoparticle and its distance from PCP. In the calculations we incorporate description of the dominant processes governing the excitation dynamics in such a hybrid nanostructure - radiative and nonradiative decay of fluorescence due to coupling with MNP, as well as energy transfer between chlorophylls. We stress that the calculations were carried out using analytical and exact description of the scattering of dipolar radiation on a spherical nanoparticle and can be extended to investigate other important features of the system, such as its quantum efficiency and lifetime of excitation. Results indicate that of all the investigated parameters, the distance between MNP and PCP is the key parameter that governs the dynamics of the system.

\section{Acknowledgments}

Financial support from the WELCOME program "Hybrid nanostructures as a stepping-stone towards efficient artificial photosynthesis" awarded by the Foundation for Polish Science is gratefully acknowledged.

\section{References}

[1] A.V. Akimov, A. Mukherjee, C.L. Yu, D.E. Chang, A.S. Zibrov, P.R. Hemmer, H. Park, M.D. Lukin, $N a-$ ture 450, 402 (2007). 
[2] A. Kinkhabwala, Z. Yu, S. Fan, Y. Avlasevich, K. Mllen, W.E. Moerner, Nature Phot. 3, 654 (2009).

[3] K.M. Mayer, J.H. Hafner, Chem. Rev. 111, 3828 (2011).

[4] H. Atwater, A. Polman, Nature Mat. 9, 205 (2010).

[5] P. Anger, P. Bharadwaj, L. Novotny, Phys. Rev. Lett. 96, 11302 (2006).

[6] H. Mertens, A. Polman, J. Appl. Phys. 105, 044302 (2009).

[7] J. Lee, A.O. Govorov, N.A. Kotov, Nano Lett. 5, 2063 (2005).

[8] A.O. Govorov, J. Lee, N.A. Kotov, Phys. Rev. B 76, 125308 (2007).

[9] M.K. Schmidt, A.O. Govorov, S. Mackowski, Mater. Res. Soc. Symp. Proc. 1286, 2011, Materials Research Society, DOI: 10.1557/opl.2011.8.

[10] I. Nabiev, A. Rakovich, A. Sukhanova, E. Lukashev, V. Zagidullin, V. Pachenko, Y. Rakovich, J.F. Donegan, A.B. Rubin, A.O. Govorov, Angew. Chem., Intl Ed. 49, 7217 (2010).

[11] S. Mackowski, S. Wormke, T.H.P. Brotosudarmo, C. Jung, R. Hiller, H. Scheer, C. Brauchle, Biophys. J. 93, 3249 (2007).

[12] A.O. Govorov, I. Carmeli, Nano Lett. 7, 620 (2007).

[13] I. Kim, S.L. Bender, J. Hranisavljevic, L.M. Utschig, L. Huang, G.P. Wiederrecht, D.M. Tiede, Nano Lett. 11, 3091 (2011).

[14] S. Mackowski, S. Wormke, A.J. Maier, T.H.P. Brotosudarmo, H. Harutyunyan, A. Hartschuh, A.O. Govorov, H. Scheer, C. Brauchle, Nano Lett. 8, 558 (2008).

[15] T.H.P. Brotosudarmo, E. Hofmann, R.G. Hiller, S. Wormke, S. Mackowski, A. Zumbusch, C. Brauchle, H. Scheer, FEBS Lett. 580, 5257 (2006).
[16] D.J. Miller, J. Catmull, R. Puskeiler, H. Tweedale, F.P. Sharples, R.G. Hiller, Photosynth. Res. 86, 229 (2005).

[17] E. Hofmann, P.M. Wrench, F.P. Sharples, R.G. Hiller, W. Welte, K. Diederichs, Science 272, 5269 (1996).

[18] F.J. Kleima, E. Hofmann, B. Gobets, I.H. van Stokkum, R. van Grondelle, K. Diederichs, H. van Amerongen, Biophys. J. 78, 344 (2000).

[19] L. Bujak, N. Czechowski, D. Piatkowski, R. Litvin, S. Mackowski, T.H.P. Brotosudarmo, R.J. Cogdell, S. Pichler, W. Heiss, Appl. Phys. Lett. 99, 173701 (2011).

[20] N. Czechowski, P. Nyga, M.K. Schmidt, T.H.P. Brotosudarmo, H. Scheer, D. Piatkowski, S. Mackowski, Plasmonics, DOI: 10.1007/s11468-011-9283-7.

[21] P.B. Johnson, R.W. Christy, Phys. Rev. B 6, 4370 (1972).

[22] X.M. Hua, J.I. Gersten, A. Nitzan, J. Chem. Phys. 83, 3650 (1985).

[23] R. Ruppin, J. Chem. Phys. 76, 1681 (1982).

[24] T. Forster, Ann. Phys. 437, 55 (1948).

[25] H.Y. Xie, H.Y. Chung, P.T. Leung, D.P. Tsai, Phys. Rev. B 80, 155448 (2009).

[26] R. Simonetto, M. Crimi, D. Sandona, R. Croce, G. Cinque, J. Breton, R. Bassi, Biochemistry 38, 12974 (1999). 